\title{
A Systematic Review of Proton Pump Inhibitors for the Prevention and Treatment of Preeclampsia and Gestational Hypertension
}

\author{
Manarangi De Silva1,2*, Fiona Brownfoot'1,2, Natalie J. Hannan ${ }^{1,2}$, Susan P. Walker ${ }^{1,2}$, \\ Catherine A. Cluver ${ }^{2.3}$, Anthea Lindquist ${ }^{2}$, Stephen Tong ${ }^{1,2}$, Roxanne Hastie ${ }^{1,2}$ \\ ${ }^{1}$ Translational Obstetrics Group, Department of Obstetrics and Gynaecology, Mercy Hospital for Women, \\ University of Melbourne, Heidelberg, Australia \\ ${ }^{2}$ Mercy Perinatal, Mercy Hospital for Women, and Department of Obstetrics and Gynecology, University of Melbourne, \\ Mercy Hospital, Heidelberg, Australia \\ ${ }^{3}$ Department of Obstetrics and Gynecology, Stellenbosch University, Cape Town, South Africa \\ Email: *rangi.desilva@unimelb.edu.au
}

How to cite this paper: De Silva, M., Brownfoot, F., Hannan, N.J., Walker, S.P., Cluver, C.A., Lindquist, A., Tong, S. and Hastie, R. (2019) A Systematic Review of Proton Pump Inhibitors for the Prevention and Treatment of Preeclampsia and Gestational Hypertension. Open Journal of $\mathrm{Ob}$ stetrics and Gynecology, 9, 21-28. https://doi.org/10.4236/ojog.2019.91003

Received: December 8, 2018

Accepted: January 1, 2019

Published: January 4, 2019

Copyright $\odot 2019$ by authors and Scientific Research Publishing Inc. This work is licensed under the Creative Commons Attribution International License (CC BY 4.0).

http://creativecommons.org/licenses/by/4.0/

\begin{abstract}
Background: Preeclampsia is a hypertensive disorder of pregnancy with a high rate of neonatal and maternal morbidity and mortality. The only definitive treatment is delivery. Through pre-clinical studies, proton pump inhibitors (PPIs), which are commonly and safely used in pregnancy, have been identified as potential therapeutic agents. Objective: To undertake a systematic review evaluating PPIs in the prevention and/or treatment of preeclampsia and gestational hypertension. Search strategy: Electronic databases were searched from inception to 2018. Search terms included preeclampsia, proton-pump inhibitors, pregnancy-induced hypertension, lansoprazole, rabeprazole, esomeprazole and omeprazole. Selection criteria: Studies were included if they were randomized control trials, case-control or cohort studies on human subjects. Case reports, review articles, opinion pieces and conference abstracts were excluded as well as studies with no or inappropriate control arms. Data collection and analysis: Only one eligible study was identified, so no analyses were able to be performed. Main results: There was only one clinical trial eligible for inclusion. This was a randomized clinical trial investigating the role of PPIs in the treatment of preterm preeclampsia in a high-risk, inpatient setting. This was a negative finding, with no change in the primary outcome of prolongation of gestation between the PPI group and placebo control. Conclusion: There is a scarcity of clinical trials published investigating the therapeutic potential of PPIs for preeclampsia and gestational hypertension. Of the one available study, PPIs were not found to pro-
\end{abstract}


long gestation among preterm preeclamptic patients compared to placebo control. In order to further elucidate the clinical potential of PPIs to prevent or treat preeclampsia, further trials are required.

\section{Keywords}

Preeclampsia, Proton-Pump-Inhibitors

\section{Introduction}

Preeclampsia is a hypertensive disorder of pregnancy, affecting $3 \%-8 \%$ of pregnant women, globally [1] [2]. It is a leading cause of maternal and neonatal morbidity and mortality, with $10 \%-15 \%$ of all maternal deaths directly associated with preeclampsia and eclampsia [3] [4]. Currently, there are no medical therapeutics available for preeclampsia and delivery is the only definite treatment, which at preterm gestations can result in significant neonatal morbidity and mortality [5] [6].

It is widely accepted that the preeclamptic placenta undergoes abnormally shallow invasion resulting in hypoxia, oxidative stress and inflammation, stimulating the release of anti-angiogenic molecules, soluble Fms-like tyrosine kinase-1 (sFLT-1) and soluble endoglin (sEng) [7] [8]. These factors enter the maternal vasculature and freely circulate, inflicting widespread endothelial dysfunction and ultimately cumulating in the clinical manifestations of disease [9] [10].

Although antihypertensives are used to reduce blood pressure and magnesium sulphate can be used to reduce the risk of eclampsia, neither are able to stop disease progression. Given this, there is a focus on identifying safe and effective therapeutics for preeclampsia. Several agents have been studied as potential therapeutics for preeclampsia. Aspirin is currently recommended for pregnant women at high risk of developing preeclampsia where it may decrease the incidence of preeclampsia by around 10\% among high risk cohorts [11] [12]. Calcium is also widely prescribed to prevent preeclampsia; however, a benefit has not been shown in calcium-replete populations [5] [13]. L-arginine, a basic amino acid precursor of nitric oxide, has also been investigated as a preventative agent. Vadilo-Ortego et al. reported L-arginine supplementation with antioxidant vitamins reduced preeclampsia in high risk women [14]. There are no other agents that have been shown to prevent preeclampsia.

Through preclinical investigation we have identified proton pump inhibitors (PPIs) as potential therapeutics for preeclampsia. PPIs are used to treat gastric reflux and importantly, are commonly used during pregnancy due to an established safety profile, with large cohort studies demonstrating PPI exposure during pregnancy does not have a detrimental effect on neonatal wellbeing [15] [16] [17]. There is some recent evidence from a meta-analysis of retrospective, cohort studies that PPIs may increase the incidence of childhood asthma [18]. However, this evidence does not support a causative effect and no change in prescribing 
practice was recommended as a result. This may need to be considered as part of large, prospective trials.

In 2017, members of our team were the first to report that PPIs may have potential as therapeutics for preeclampsia [7] [8]. In laboratory studies we demonstrated that PPIs significantly reduced the secretion of two anti-angiogenic factors that may be driving the pathogenesis of preeclampsia, sFLT-1 and sEng. Additionally, PPIs rescued endothelial dysfunction in-vitro and ex vivo and significantly reduced blood pressure in a mouse model of preeclampsia. Furthermore, a secondary analysis of a prospective cohort study of 430 women supported these findings, where it was found that sFLT-1 and sEng levels were lower among women with confirmed or suspected preeclampsia who were coincidentally taking PPIs, compared with non-PPI users [19].

Given these findings, we aimed to investigate the clinical evidence for the therapeutic role of PPIs for preeclampsia via a systematic review of the current literature.

\section{Objective}

To undertake a systematic review evaluating the use of PPIs during pregnancy and the development of hypertensive disorders, including preeclampsia and gestational hypertension.

\section{Methods}

The review protocol for this study is registered through PROSPERO (CRD 42018109410). The study followed Preferred Reporting Items for Systematic Review and Meta-analyses (PRISMA) guidelines.

\subsection{Search Strategy}

We used a predefined, computer-based search strategy including the search terms proton pump inhibitors, preeclampsia, hypertension, pregnancy induced, lansoprazole/omeprazole/esomeprazole/rabeprazole. The search term "preeclampsia" was used first, followed by "proton-pump inhibitors", followed by "hypertension, pregnancy induced", and then "lansoprazole/omeprazole/ esomeprazole or rabeprazole". We then combined searches 2 and 4, followed by 1 and 3 and the results of these secondary searches were finally combined. We used this search strategy in MEDLINE, EMBASE, OVID and Cochrane databases up to October 2018.

\subsection{Inclusion Criteria and Selection}

Studies were chosen to be included if they were randomized control trials, case-control or cohort studies. Case reports, review articles, opinion pieces and conference abstracts were excluded as well as studies with no control or inappropriate control groups. Preclinical studies and studies in languages other than English were also excluded. 


\subsection{Data Extraction}

For each included article, 2 reviewers (MD and $\mathrm{RH}$ ) planned to extract data on both clinical and methodologic study characteristics using a standardised data extraction form, with any dispute resolved by a third reviewer (FB). However, as only one study was identified to be eligbible this was not possible and a narrative review of this study was performed. The quality of study and assessment of bias was performed using the RoB 2.0 tool [20]. The tool is structured into five domains through which bias might be introduced into the result and each domain has signaling questions designed to assess the risk of bias. The five domains are; bias arising from randomization, bias due to deviations from intended interventions, bias due to missing outcome data, bias in measurement of the outcome, bias in selection of the reported result. The responses of each signaling question may be "no", "probably no", "yes", "probably yes", or "no information". From this, each domain is judged as having "low risk", "some concerns", or "high risk".

\section{Results}

Of six studies initially identified in our search, five were ineligible, leaving one remaining study eligible for qualitative synthesis (Figure 1). Of the five that were ineligible, three were excluded as they investigated the effect of PPIs on endothelial markers in vitro, one study investigated the effect of esomeprazole compared to magnesium sulphate using preeclamptic rats and the last was a review of the treatment of reflux disease in pregnancy and lactation. The remaining study that was eligible for inclusion in the analysis investigated PPIs in a randomized placebo-controlled trial among women with preterm preeclampsia. Given there is only one study that was considered eligible for inclusion in this meta-analysis according to our prespecified inclusion criteria, we were unable to proceed to data extraction and meta-analysis. Instead, we will describe the findings of the eligible study.

Cluver et al. recently published their study evaluating whether oral esomeprazole can prolong pregnancies complicated by preterm preeclampsia [21]. This was a double-blinded, placebo-controlled, randomized trial based in South Africa, where the incidence of preeclampsia is higher than in high income countries [5]. Women with preterm preeclampsia were assigned to $40 \mathrm{mg}$ of daily esomeprazole, or an identical placebo. The primary outcome was prolongation of pregnancy by 5 days, and secondary maternal and neonatal outcomes were also reported. There was no change in the primary outcome between the groups (median 11.4 days in the esomeprazole group vs 8.3 days in the placebo group), however there was a non-significant trend in prolongation by 3 days in the esomeprazole group. No difference was found in sFLT1 and sEng concentrations between groups. There were also no significant differences in secondary maternal, fetal, and neonatal outcomes, except for placental abruption, however this was not significant after adjustment [21]. Using the RoB 2.0 tool [20], we assessed this single study for risk of bias and a judgement of low risk of bias across all domains was found (Table 1). 


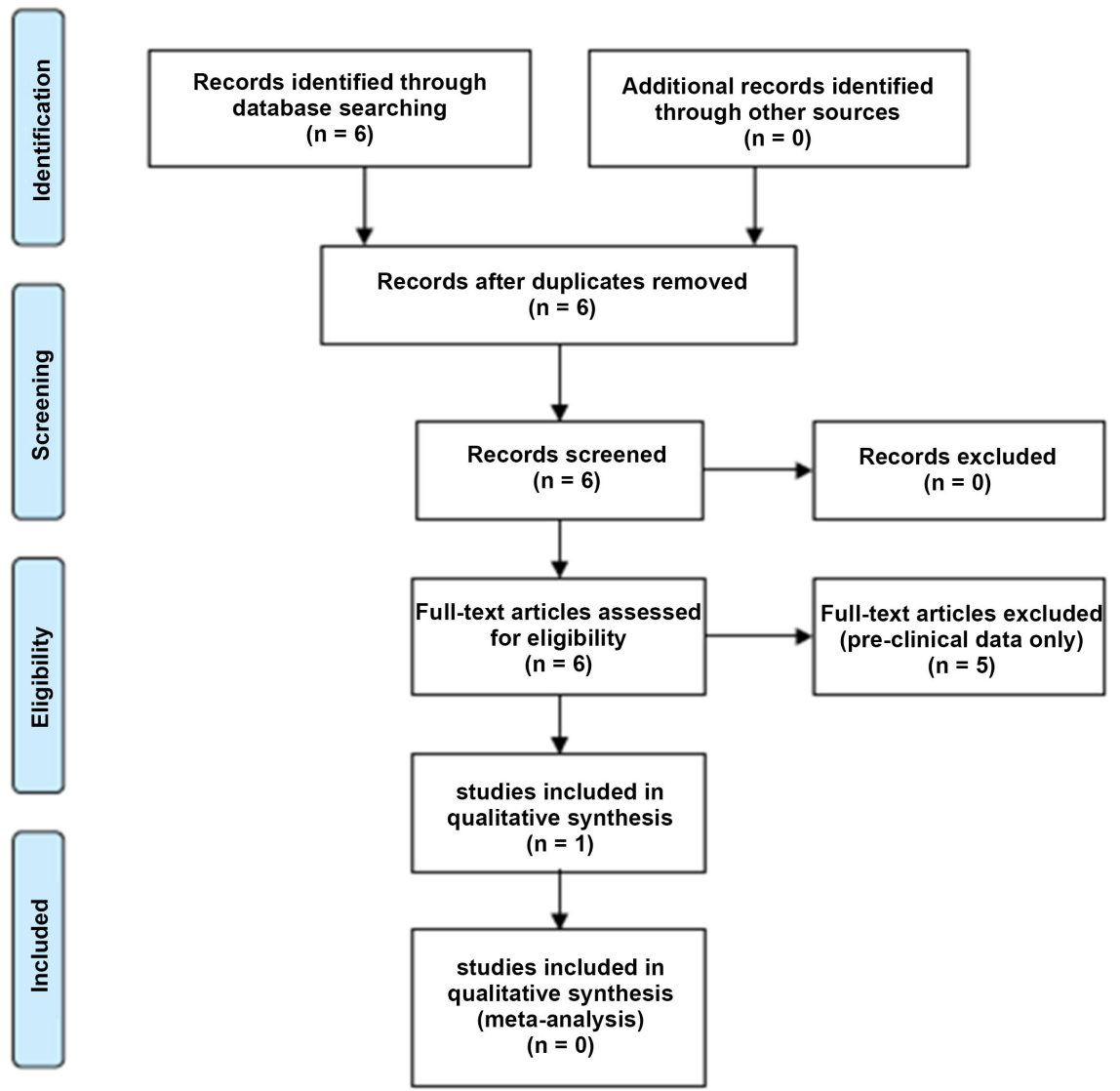

Figure 1. PRISMA Flow diagram.

Table 1. RoB 2.0 tool risk of bias assessment for Cluver et al. [21].

\begin{tabular}{cl}
\hline Signaling Questions & Response \\
\hline Bias arising from the randomization process & Low risk \\
Bias due to deviations from intended interventions & Low risk \\
Bias due to missing outcome data & Low risk \\
Bias in measurement of the outcomes & Low risk \\
Bias in selection of the reported results & Low risk \\
\hline
\end{tabular}

\section{Discussion}

There is laboratory evidence [7] [8] and data from observational studies to [19] suggest PPIs may have a role as a therapeutic agent in the prevention and/or treatment of preeclampsia.

This systematic meta-analysis only identified one clinical trial investigating PPIs as a treatment for preeclampsia. No studies evaluating the potential of PPIs to prevent preeclampsia were identified. Five other studies were excluded as they did not meet inclusion criteria for this review. Although the study we identified was recently completed and the risk of bias was low, the study was small and unable to demonstrate a significant effect on prolongation of pregnancy when PPIs were administered to preterm preeclamptic patients. There was, however, a 
non-significant trend towards reduced incidence of placental abruption among women treated with PPIs. The authors hypothesized that the negative finding may have been secondary to decreased circulating levels of esomeprazole due to the severity of proteinuria and suggest a higher dose of esomeprazole may be required to demonstrate a significant effect in this cohort.

The lack of studies identified in this review may be attributed to the fact that the investigation of PPIs as a therapeutic agent against preeclampsia is fairly new. However, despite a lack of clinical evidence in this area, reviews which include only one study, or even empty reviews, identify gaps in knowledge and provide important direction for future, targeted research [22] [23]. At the time of this review, there is a paucity of clinical data to support the preclinical findings detailed above and it is difficult to draw conclusions from the single trial included. There is one ongoing trial based in Egypt named the ESOPE trial (NCT03213639), which aims to investigate the role of esomeprazole for the treatment of early onset preeclampsia. There are two other trials registered that will investigate the role of PPIs in preventing preeclampsia and have a planned commencement date of 2019. A phase II clinical trial named ESPRESSO (ACTRN12618001755224) aims to administer $40 \mathrm{mg}$ of esomeprazole or placebo to women identified at higher risk of developing preeclampsia. The primary outcome for this study is mean-arterial pressure at 36 weeks gestation. A larger phase III clinical trial will also be commencing in 2019, the APPLE PIE study (ACTRN12618000690257p), which is investigating the role of esomeprazole in 5500 obese, nulliparous women with the primary outcome being the incidence of preeclampsia.

The present analysis is significantly limited by a lack of existing research despite significant laboratory data. This systematic review should be updated when more studies are available in this area.

\section{Conclusions}

There is only one randomized controlled trial available investigating the potential of PPIs in the treatment of preeclampsia.

From this review, we observed that there is a paucity of clinical evidence. PPIs remain a promising therapeutic candidate given the strong preclinical data, therefore further investigations of PPIs are required in order to fully elucidate the clinical role of PPIs in the prevention and/or treatment of preeclampsia and any potential risks involved.

\section{Funding}

R. Hastie was supported by a Melbourne Medical School, Department of Obstetrics and Gynaecology Fellowship, S. Tong (\#1136418), F. Brownfoot (\#1142636) and N. J Hannan (\#1146128) were supported by NHMRC Fellowships.

\section{Conflicts of Interest}

The authors declare no conflicts of interest regarding the publication of this paper. 


\section{References}

[1] Hofmeyr, G.J., Lawrie, T.A., Atallah, A.N., Duley, L. and Torloni, M.R. (2014) Calcium Supplementation during Pregnancy for Preventing Hypertensive Disorders and Related Problems. Cochrane Database of Systematic Reviews, 6, CD001059.

[2] Rolnik, D., Wright, D., Poon, L., et al. (2017) Aspirin vs Placebo in Pregnancies at High Risk of Preterm Preeclampsia. The New England Journal of Medicine, 377, 613-622. https://doi.org/10.1056/NEJMoa1704559

[3] Duley, L., Henderson-Smart, D.J., Meher, S. and King, J.F. (2007) Antiplatelet Agents for Preventing Pre-Eclampsia and Its Complications. Cochrane Database of Systematic Reviews, CD004659.

[4] Brown, M., Magee, L., Kenny, L., et al. (2018) Hypertensive Disorders of Pregnancy: ISSHP Classification, Diagnosis \& Management Recommendations for International Practice. Pregnancy Hypertension, 13, 291-310.

[5] Sibai, B., Dekker, G. and Kupferminc, M. (2005) Pre-Eclampsia. Lancet, 365, 785-799. https://doi.org/10.1016/S0140-6736(05)71003-5

[6] Magee, L.A., Pels, A., Helewa, M., et al. (2014) Diagnosis, Evaluation, and Management of the Hypertensive Disorders of Pregnancy. Pregnancy Hypertension, 4, 105-145. https://doi.org/10.1016/j.preghy.2014.01.003

[7] Onda, K., Tong, S., et al. (2017) Proton Pump Inhibitors Decrease sFlt-1 and Soluble Endoglin Secretion, Decrease Hypertension and Rescue Endothelial Dysfunction. Hypertension, 69, 457-468. https://doi.org/10.1161/HYPERTENSIONAHA.116.08408

[8] Onda, K., Hannan, N., Beard, S., et al. (2014) Proton Pump Inhibitors for Treatment of Preeclampsia. Abstract 6-OR. XIX Word Congress ISSHP, 26-29 October 2014.

[9] Veerareddy, S., Cooke, C., Baker, P. and Davidge, S. (2002) Vascular Adaptation to Pregnancy in Mice: Effects on Myogenic Tone. American Journal of Physiology-Heart and Circulatory Physiology, 283, 2226-2233. https://doi.org/10.1152/ajpheart.00593.2002

[10] Young, B., Levine, R. and Ananth Karamanchi, S. (2010) Pathogenesis of Preeclampsia. Annual Review of Pathology: Mechanisms of Disease, 5, 173-192. https://doi.org/10.1146/annurev-pathol-121808-102149

[11] Hutcheon, J.A., Lisonkova, S. and Joseph, K.S. (2011) Epidemiology of Pre-Eclampsia and the Other Hypertensive Disorders of Pregnancy. Best Practice \& Research Clinical Obstetrics \& Gynaecology, 25, 391-403. https://doi.org/10.1016/j.bpobgyn.2011.01.006

[12] Askie, L., Duley, L., Henderson-Scott, D.L. and Stewart, L.A. (2007) Antiplatelet Agents for Prevention of Preeclampsia: A Metalaysis of Individual Patient Data. Lancet, 269, 1791-1798. https://doi.org/10.1016/S0140-6736(07)60712-0

[13] Redman, C.W. and Sargent, I.L. (2005) Latest Advances in Understanding Preeclampsia. Science, 308, 1592-1594. https://doi.org/10.1126/science.1111726

[14] Vadillo-Ortego, F., Perihcart-Perera, O., Espino, S., et al. (2011) Effect of Supplementation during Pregnancy of L-Arginine and Antioxidant Vitamins in Medical Food on Preeclampsia in High Risk Population: Randomized Controlled Trial. BMJ, 342, d2901. https://doi.org/10.1136/bmj.d2901

[15] Gill, S.K., O’Brien, L., Einarson, T.R. and Koren, G. (2009) The Safety of Proton Pump Inhibitors (PPIs) in Pregnancy: A Meta-Analysis. The American Journal of Gastroenterology, 104, 1541-1545; quiz 1540, 1546. 
[16] Pasternak, B. and Hviid, A. (2010) Use of Proton-Pump Inhibitors in Early Pregnancy and the Risk of Birth Defects. The New England Journal of Medicine, 363, 2114-2123. https://doi.org/10.1056/NEJMoa1002689

[17] Matok, I., Levy, A., Wiznitzer, A., Uziel, E., Koren, G. and Gorodischer, R. (2012) The Safety of Fetal Exposure to Proton-Pump Inhibitors during Pregnancy. Digestive Diseases and Sciences, 57, 699-705. https://doi.org/10.1007/s10620-011-1940-3

[18] Lai, T., Wu, M., Liu, J., et al. (2018) Acid-Suppressive Drug Use during Pregnancy and the Risk of Childhood Asthma: A Meta-Analysis. Pediatrics, 141, e20170889. https://doi.org/10.1542/peds.2017-0889

[19] Saleh, L., Samantar, R., Garrelds, I., et al. (2017) Low Soluble Fms-Like Tyrosine Kinase-1, Endoglin, and Endothelin-1 Levels in Women with Confirmed or Suspected Preeclampsia Using Proton Pump Inhibitors. Hypertension, 70, 594-600.

[20] Higgins, J.P.T., Sterne, J.A.C., Savović, J., Page, M.J., Hróbjartsson, A., Boutron, I., Reeves, B. and Eldridge, S. (2016) A Revised Tool for Assessing Risk of Bias in Randomized Trials. In: Chandler, J., McKenzie, J., Boutron, I. and Welch, V., Cochrane Methods. Cochrane Database of Systematic Reviews, Issue 10 (Suppl 1).

[21] Cluver, C., Hannan, N., van Papendorp, E., et al. (2018) Esomeprazole to Treat Women with Preterm Preeclampsia: A Randomized Placebo Controlled Trial. AJOG, 219, 388 e1-e17.

[22] Yaffe, J., Montgomery, P., Hopewell, S. and Shepard, L.D. (2012) Empty Reviews: A Description and Consideration of Cochrane Systematic Reviews with no Included Studies. PLoS ONE, 7, e36626. https://doi.org/10.1371/journal.pone.0036626

[23] Prasthanti, E., Sumanth, K.N., Renjith George, P., Karanth, L. and Soe, H.H.K. (2015) Management of Gag Reflex for Patients Undergoing Dental Treatment. Cochrane Database of Systematic Reviews, 10, C 011116. 